\title{
Unidirectional spin Hall magnetoresistance in ferromagnet/normal metal bilayers
}

\author{
Can Onur Avci ${ }^{\star}$, Kevin Garello, Abhijit Ghosh, Mihai Gabureac, Santos F. Alvarado \\ and Pietro Gambardella*
}

\begin{abstract}
Magnetoresistive effects are usually invariant on inversion of the magnetization direction. In non-centrosymmetric conductors, however, nonlinear resistive terms can give rise to a current dependence that is quadratic in the applied voltage and linear in the magnetization. Here we demonstrate that such conditions are realized in simple bilayer metal films where the spin-orbit interaction and spin-dependent scattering couple the current-induced spin accumulation to the electrical conductivity. We show that the longitudinal resistance of $\mathrm{Ta} \mid \mathrm{Co}$ and $\mathrm{Pt} \mid \mathrm{Co}$ bilayers changes when reversing the polarity of the current or the sign of the magnetization. This unidirectional magnetoresistance scales linearly with current density and has opposite sign in $\mathrm{Ta}$ and $\mathrm{Pt}$, which we associate with the modification of the interface scattering potential induced by the spin Hall effect in these materials. Our results suggest a route to control the resistance and detect magnetization switching in spintronic devices using a two-terminal geometry, which applies also to heterostructures including topological insulators.
\end{abstract}

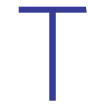
he effects of the magnetization on the electric conductivity of metals have been studied for a long time ${ }^{1}$, providing understanding of fundamental phenomena associated with electron transport and magnetism as well as a multitude of applications in sensor technology. The anisotropic magnetoresistance (AMR) - the change of the resistance of a material on rotation of the magnetization-is a prominent manifestation of spin-orbit coupling and spin-dependent conductivity in bulk ferromagnets ${ }^{2,3}$. In thin-film heterostructures, the additional possibility of orienting the magnetization of stacked ferromagnetic layers parallel or antiparallel to each other gives rise to the celebrated giant magnetoresistance (GMR) effect ${ }^{4,5}$, which has played a major role in all modern developments of spintronics. Together with the early spin-injection experiments $^{6,7}$, the study of GMR revealed how non-equilibrium spin accumulation at the interface between ferromagnetic (FM) and normal metal (NM) conductors governs the propagation of spin currents $^{8-11}$ and, ultimately, the conductivity of multilayer systems ${ }^{10,12}$.

Recently, it has been shown that significant spin accumulation at a FM/NM interface can be achieved using a current-in-plane (CIP) geometry owing to the spin Hall effect (SHE) in the NM (ref. 13). When the FM is a metal and NM is a heavy element such as Pt or $\mathrm{Ta}$, the spin accumulation is strong enough to induce magnetization reversal of nanometre-thick FM layers at current densities of the order of $j=10^{7}-10^{8} \mathrm{~A} \mathrm{~cm}^{-2}$ (refs 14-16). When the FM is an insulator, such as yttrium iron garnet, the SHE causes an unusual magnetoresistance associated with the back-flow of a spin current into the NM when the spin accumulation $\mu_{\mathrm{s}} \sim(\mathbf{j} \times \hat{\mathbf{z}})$ is aligned with the magnetization of the FM, which increases the conductivity of the NM due to the inverse SHE (refs 17-20). This so-called spin Hall magnetoresistance (SMR) is characterized by $R^{y}<R^{z} \approx R^{x}$, where $R^{i}$ is the resistance measured when the magnetization $(\mathbf{M})$ is saturated parallel to $i=x, y, z$, and differs from the conventional AMR in polycrystalline samples, for which $R=R^{x}-\left(R^{y, z}-R^{x}\right)[\hat{\mathbf{M}} \cdot \hat{\mathbf{j}}]^{2}$ and $R^{y} \approx R^{z}<R^{x}$ (ref. 3).

In this work, we report on a magnetoresistance effect occurring in FM/NM bilayers with the NM possessing a large SHE. The effect combines features that are typical of the current-in-plane (CIP) GMR and SHE, whereby the spin accumulation induced by the SHE in the NM replaces one of the FM polarizers of a typical GMR device. Differently from GMR, however, this effect introduces a nonlinear dependence of the resistance on the current, which gives it unique unidirectional properties: the resistivity changes when reversing either the sign of the magnetization or the polarity of the current, increasing (decreasing) when the SHE-induced nonequilibrium magnetization at the $\mathrm{FM} / \mathrm{NM}$ interface is oriented parallel (antiparallel) to the magnetization of the FM, as illustrated in Fig. 1a,b. We associate this phenomenon with the modulation of the FM/NM interface resistance due to the SHE-induced spin accumulation, which gives rise to a nonlinear contribution to the longitudinal conductivity that scales proportionally with the current density and has opposite sign in Pt and Ta. Contrary to the linear magnetoresistive effects, including the AMR, GMR, and SMR described above, which are even with respect to the inversion of either the current or magnetization owing to the time reversal symmetry embodied in the Onsager's reciprocity relations, here we observe $R(\mathbf{j}, \mathbf{M})=-R(-\mathbf{j}, \mathbf{M})=-R(\mathbf{j},-\mathbf{M})$, providing a unidirectional contribution to the magnetoresistance in simple bilayer systems.

\section{Sample layout}

The samples studied in this work are Pt $(1-9 \mathrm{~nm}) \mid$ Co $(2.5 \mathrm{~nm})$ and $\mathrm{Ta}(1-9 \mathrm{~nm}) \mid \mathrm{Co}(2.5 \mathrm{~nm})$ films with spontaneous in-plane magnetization, capped by $2 \mathrm{~nm}$ of $\mathrm{AlO}_{x}$ and patterned in the shape of Hall bars of nominal length $l=20-50 \mu \mathrm{m}$, width $w=4-10 \mu \mathrm{m}$, and $l / w=4$, as shown in Fig. 1c. Additional control experiments were carried out on single $\mathrm{Co}$, Ta and Pt films, and $\mathrm{Ta}(1,6 \mathrm{~nm}) \mid \mathrm{Cu}$ $(2,4,6 \mathrm{~nm}) \mid$ Co $(2.5 \mathrm{~nm})$ trilayers. To characterize the magnetic and electrical properties of these layers we performed harmonic measurements of the longitudinal resistance $(R$, see Supplementary Information) and transverse Hall resistance $\left(R_{\mathrm{H}}\right.$; refs $\left.16,21-23\right)$ as a function of a vector magnetic field defined by the polar and azimuthal coordinates $\theta_{\mathrm{B}}$ and $\varphi_{\mathrm{B}}$. The measurements were carried 


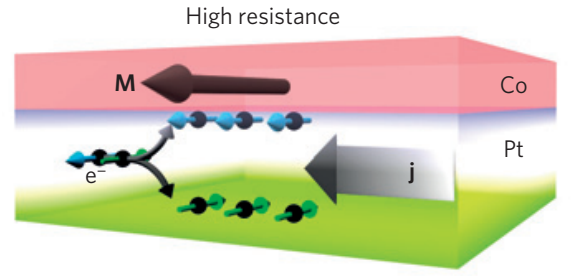

b

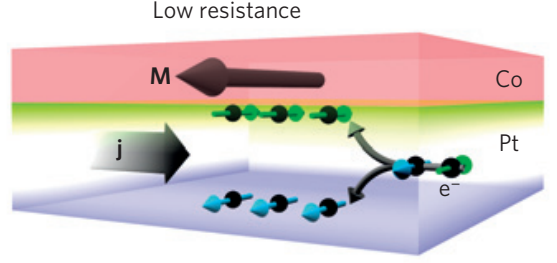

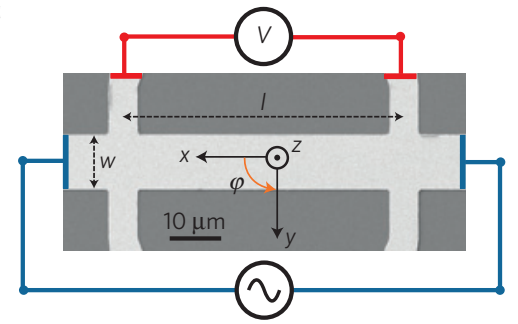

Figure 1 | Illustration of the unidirectional spin Hall magnetoresistance effect and sample layout. a, Parallel alignment of the SHE-induced non-equilibrium magnetization at the FM/NM interface with the magnetization of the FM increases the resistivity of the bilayer. $\mathbf{b}$, Antiparallel alignment decreases the resistivity. The arrows in $\mathbf{a}, \mathbf{b}$ indicate the direction of the spin magnetic moment. $\mathbf{c}$, Scanning electron micrograph of a sample and schematics of the longitudinal resistance measurements.

a
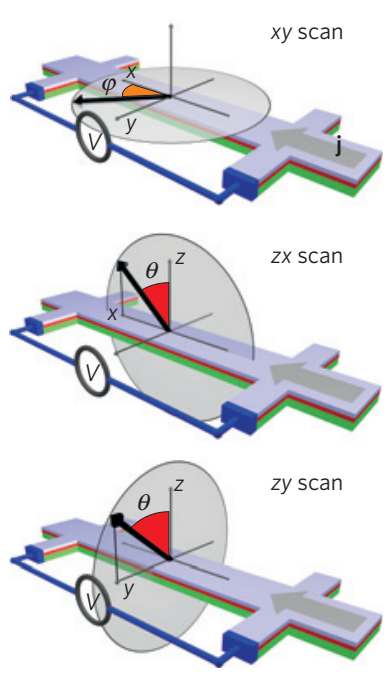

b

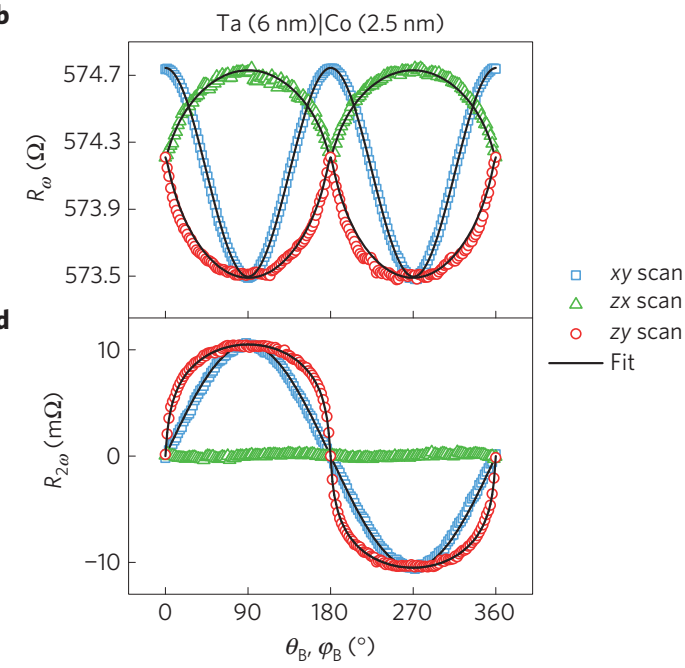

c

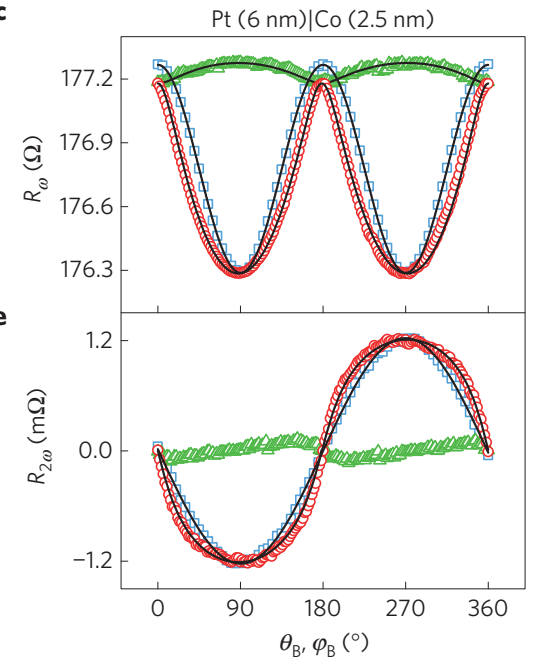

Figure 2 | Linear and nonlinear magnetoresistance. a, Geometry of the measurements. b,c, First-harmonic resistance of Ta (6 nm)|Co (2.5 nm) (c) and Pt $(6 \mathrm{~nm}) \mid \mathrm{Co}(2.5 \mathrm{~nm})(\mathbf{c})$, measured with a current density of $j=10^{7} \mathrm{~A} \mathrm{~cm}^{-2}$. d,e, Second-harmonic resistance measured simultaneously with $\mathbf{b}, \mathbf{c}$. The dimensions of the Hall bars are $I=50 \mu \mathrm{m}$ and $w=10 \mu \mathrm{m}$.

out at room temperature by injecting an a.c. current of frequency $\omega / 2 \pi=10 \mathrm{~Hz}$ and simultaneously recording the first $\left(R_{\omega}\right)$ and second harmonic resistance $\left(R_{2 \omega}\right)$ between the contacts shown in Fig. 1c while rotating the sample in a uniform magnetic field of $1.7 \mathrm{~T}$. Here, $R_{\omega}$ represents the linear response of the samples to the current-that is, the conventional resistance. To include the different magnetoresistive angular dependencies in a single expression we write this term as

$$
R_{\omega}=R^{z}+\left(R^{x}-R^{z}\right) \sin ^{2} \theta \cos ^{2} \varphi+\left(R^{y}-R^{z}\right) \sin ^{2} \theta \sin ^{2} \varphi
$$

where $\theta$ and $\varphi$ are the polar and azimuthal angles of $\mathbf{M}$, as schematized in Fig. 2a. $R_{2 \omega}$ describes resistance contributions that vary quadratically with the applied voltage and includes the currentinduced changes of resistivity that are the main focus of this work.

\section{Magnetoresistance measurements}

Figure $2 \mathrm{~b}, \mathrm{c}$ shows the resistance of $\mathrm{Ta}(6 \mathrm{~nm}) \mid \mathrm{Co}(2.5 \mathrm{~nm})$ and $\mathrm{Pt}$ $(6 \mathrm{~nm}) \mid \mathrm{Co}(2.5 \mathrm{~nm})$ layers during rotation of the applied field in the $x y, z x$ and $z y$ planes. We observe a sizeable magnetoresistance (MR) in all three orthogonal planes and $R^{x}>R^{z}>R^{y}$ for both samples, in agreement with previous measurements on $\mathrm{Pt} \mid \mathrm{Co}$ films ${ }^{24,25}$. The resistivity of $\mathrm{Ta}(6 \mathrm{~nm}) \mid \mathrm{Co}(2.5 \mathrm{~nm})[\mathrm{Pt}(6 \mathrm{~nm}) \mid \mathrm{Co}(2.5 \mathrm{~nm})]$ is 108.9 (36.8) $\mu \Omega \mathrm{cm}$ and the MR ratios are $\left(R^{x}-R^{z}\right) / R^{z}=0.09 \%[0.05 \%]$ and $\left(R^{z}-R^{y}\right) / R^{z}=0.12 \%[0.53 \%]$, showing significant SMR-like behaviour, a factor 10 to 100 times larger compared to Ta|YIG and Pt|YIG (refs 17,18). The solid lines represent fits to the MR using equation (1) and $\theta$ simultaneously measured via the anomalous Hall resistance (see Supplementary Information).

As well as the linear resistance, we measure an unexpected nonlinear resistance, $R_{2 \omega}$, which has a different angular dependence from that of $R_{\omega}$ and opposite sign in Pt and Ta, as shown in Fig. 2d,e. By fitting the curves with respect to the angles $\theta$ and $\varphi$ (solid lines), we find that $R_{2 \omega} \sim \sin \theta \sin \varphi \sim M_{y}$. In the following, we discuss the types of nonlinear effects that can give rise to such a symmetry.

\section{Spin-orbit torques and thermoelectric contributions}

First, we consider oscillations of the magnetization due to the current-induced spin-orbit torques (SOTs; refs 16,21-23,26). As the SOTs are proportional to the current, a.c. oscillations of the magnetization can introduce second-order contributions to $R_{2 \omega}$ due to the first-order MR described by equation (1). However, as shown in detail in the Supplementary Information, the SOT-induced signal is not compatible with the angular dependence of $R_{2 \omega}$. Both the fieldlike and antidamping-like SOT (as well as the torque due to the Oersted field) vanish for $\mathbf{M} \| y$, where $\left|R_{2 \omega}\right|$ is maximum. Moreover, the field-like SOT is small in 2.5-nm-thick Co layers ${ }^{23}$, whereas the antidamping SOT can only induce variations of $R_{2 \omega}$ in the $z x$ plane with maxima and minima close to $\theta_{\mathrm{B}}=0^{\circ}$ and $180^{\circ}$, which we observe to be small and more pronounced in $\mathrm{Pt} \mid \mathrm{Co}$ relative to $\mathrm{Ta} \mid \mathrm{Co}$ (Fig. 2d,e).

Second, we analyse the influence of thermal gradients $(\nabla T)$ and related thermoelectric effects. The anomalous Nernst effect (ANE) and spin Seebeck effect (SSE; refs 27,28), both inducing a 

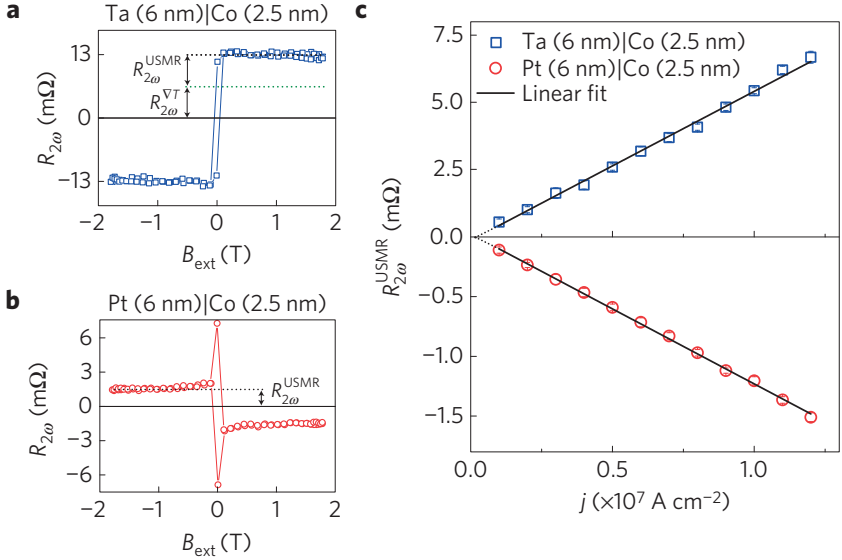

Figure 3 | Field and current dependence of the nonlinear magnetoresistance. a,b, $R_{2 \omega}$ of $\mathrm{Ta}(6 \mathrm{~nm}) \mid \mathrm{Co}(2.5 \mathrm{~nm})(\mathbf{a})$ and $\mathrm{Pt}$ $(6 \mathrm{~nm}) \mid \mathrm{Co}(2.5 \mathrm{~nm})(\mathbf{b})$ recorded during a field sweep along $y$ with a current density of $j=1.2 \times 10^{7} \mathrm{~A} \mathrm{~cm}^{-2}$. c, Current dependence of $R_{2 \omega}^{\cup S M R}$. The solid lines are fits to the data. The slope gives the amplitude of the USMR, which is $5.5 \mathrm{~m} \Omega$ and $1.25 \mathrm{~m} \Omega$ per $10^{7} \mathrm{~A} \mathrm{~cm}^{-2}$ for these samples, respectively. The thermal contribution $R_{2 \omega}^{\nabla T}$ has been subtracted from the TalCo data. The dimensions of the Hall bars are $I=50 \mu \mathrm{m}$ and $w=10 \mu \mathrm{m}$.

longitudinal voltage proportional to $j^{2}(\mathbf{M} \times \nabla T)$, can give rise to a similar angular dependence as observed for $R_{2 \omega}$ when $\nabla T \| \hat{\mathbf{z}}$ (see Supplementary Information). Here, we find that thermoelectric voltages are negligible in $\mathrm{Pt} \mid \mathrm{Co}$, in agreement with the very small thermal gradients reported for this system ${ }^{23}$. In $\mathrm{Ta} \mid \mathrm{Co}$, on the other hand, the much larger resistivity of Ta relative to Co results in a higher current flowing in the Co layer and a positive $\nabla T$. In such a case, the second harmonic signal of thermal origin, $R_{2 \omega}^{\nabla T}$, can be simply estimated from its transverse (Hall) counterpart scaled by the geometric factor $l / w$ when the magnetization is tilted in the $x$ direction, and subtracted from the raw $R_{2 \omega}$ signal. Accordingly, we find that $R_{2 \omega}^{\nabla T}=5 \mathrm{~m} \Omega$ in Ta $(6 \mathrm{~nm}) \mid$ Co $(2.5 \mathrm{~nm})$, which accounts for only about $50 \%$ of the total $R_{2 \omega}$ reported in Fig. 2. The same procedure applied to Pt|Co gives $R_{2 \omega}^{\nabla T}$ of the order of $5 \%$ of the total $R_{2 \omega}$, whereas in the control samples lacking a heavy metal we find uniquely a signal of thermal (ANE) origin. We conclude that there is an additional magnetoresistive effect in the Pt|Co and $\mathrm{Ta} \mid \mathrm{Co}$ bilayers that cannot be accounted for by either current-induced magnetization dynamics or thermoelectric voltages.

\section{Unidirectional spin Hall magnetoresistance}

The symmetry as well as the opposite sign of the nonlinear resistance in $\mathrm{Ta} \mid \mathrm{Co}$ and $\mathrm{Pt} \mid \mathrm{Co}$ suggest that it is related to the scalar product of the magnetization with the SHE-induced spin accumulation at the FM/NM interface, $(\mathbf{j} \times \hat{\mathbf{z}}) \cdot \mathbf{M}$, giving rise to a chiral MR contribution $R_{2 \omega}^{\mathrm{USMR}} \sim \mathbf{j} \times \mathbf{M}$. This relation describes the general features expected from a unidirectional magnetoresistance driven by the spin Hall effect (USMR). We note that this MR contribution depends on the current direction and that the resistance of the bilayer increases when the direction of the majority spins in the FM and the spin accumulation vector are parallel to each other, and decreases when they are antiparallel. This may seem counterintuitive at first sight, considering that the conductivity of Co is larger for the majority spins. However, as we will discuss later, this behaviour is consistent with the theory of GMR in FM|NM|FM heterostructures ${ }^{10,29,30}$ when only a single FM/NM interface is retained and the SHE is taken into account.

To investigate further the USMR we have measured $R_{2 \omega}$ as a function of an external magnetic field applied parallel to $\hat{\mathbf{y}}$ and a
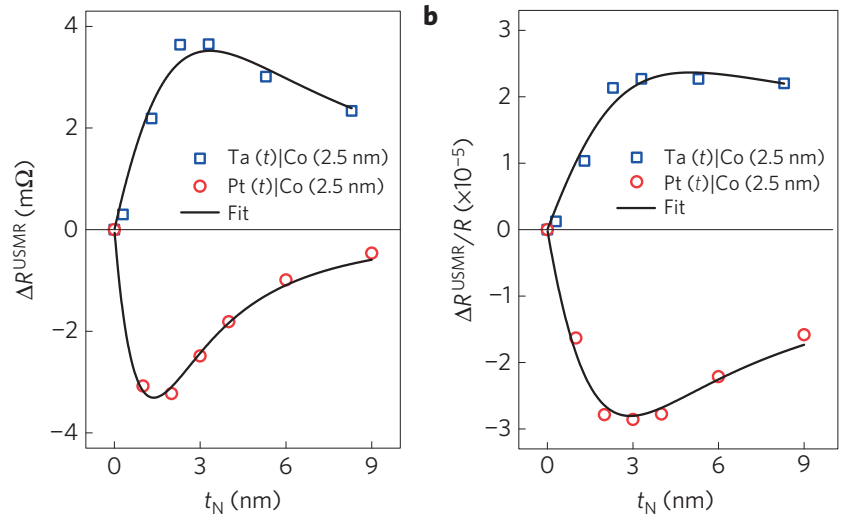

Figure 4 | USMR as a function of NM thickness. a, Sheet resistance $\Delta R^{\text {USMR }}$ as a function of Ta (squares) and Pt (circles) thickness measured at constant current density $j=10^{7} \mathrm{~A} \mathrm{~cm}^{-2}$. The Co layer is $2.5 \mathrm{~nm}$ thick in all samples. b, Normalized resistance $\Delta R^{U S M R} / R$. The solid lines are fits to the data according to the model described in the text.

current amplitude. Figure 3 shows that $R_{2 \omega}$ is constant as a function of field for Ta|Co (Fig. 3a) as well as for Pt|Co (Fig. 3b) and reverses sign upon switching the magnetization from the $y$ to the $-y$ direction. In the $\mathrm{Pt}$ |Co case we observe also two spikes, which we attribute to the magnetization breaking into domains at low field and giving rise to dynamic effects on the domain walls ${ }^{16}$. Note that the magnetization of $\mathrm{Pt} \mid \mathrm{Co}$ is not fully saturated below $0.65 \mathrm{~T}$ due to the large perpendicular magnetic anisotropy of this system, differently from $\mathrm{Ta} \mid \mathrm{Co}$ (Supplementary Information). Figure $3 \mathrm{c}$ shows the current dependence of $R_{2 \omega}^{\mathrm{USMR}}=R_{2 \omega}-R_{2 \omega}^{\nabla T}\left(R_{2 \omega}^{\mathrm{USMR}} \approx R_{2 \omega}\right.$ for $\mathrm{Pt} \mid \mathrm{Co}$ ) obtained by taking the average of the data measured at fields larger than $| \pm 1|$ T. $R_{2 \omega}^{\text {USMR }}$ is linear with the injected current density and converges to zero within the error bar of the linear fit (black lines).

To verify the role of the interfacial spin accumulation due to the SHE we examined the dependence of the USMR on the thickness of the NM. Figure 4a,b shows the absolute change of sheet resistance $\Delta R^{\mathrm{USMR}}=R_{2 \omega}^{\mathrm{USMR}}( \pm \mathbf{M}, \pm \mathbf{j})-R_{2 \omega}^{\mathrm{USMR}}( \pm \mathbf{M}, \mp \mathbf{j})$ and the relative change of resistivity $\Delta R^{\mathrm{USMR}} / R$ measured at constant current density as a function of the $\mathrm{Ta}$ and Pt thickness. Both curves exhibit qualitatively similar behaviour: an initial sharp increase below $2-3 \mathrm{~nm}$ and a gradual decrease as the NM layer becomes thicker. We note that the USMR signal is almost absent in $\mathrm{Ta}(1 \mathrm{~nm}) \mid \mathrm{Co}$, contrary to $\mathrm{Pt}(1 \mathrm{~nm}) \mid \mathrm{Co}$, which we attribute to the oxidation of $\mathrm{Ta}$ when deposited on $\mathrm{SiO}_{2}$ and its consequent poor contribution to electrical conduction. The initial increase of the USMR is consistent with the increment of the spin accumulation at the FM/NM interface as the thickness of the NM becomes larger than the spin diffusion length, which is of the order of $1.5 \mathrm{~nm}$ in both $\mathrm{Ta}$ and $\mathrm{Pt}$ (refs 18,31). Moreover, we observe that the decline of the signal in the thicker samples is stronger in $\mathrm{Pt} \mid \mathrm{Co}$ than in $\mathrm{Ta} \mid \mathrm{Co}$. This behaviour is consistent with $\mathrm{Pt}$ gradually dominating the conduction due to its low resistivity, and a smaller proportion of the current experiencing interface scattering in $\mathrm{Pt} \mid \mathrm{Co}$. Conversely, the high resistivity of Ta shunts the current towards the Co side, increasing the relative proportion of the current affected by scattering at the $\mathrm{Ta} /$ Co interface.

As an additional check to validate these arguments we have performed measurements on single $\mathrm{Ta}(6 \mathrm{~nm}), \mathrm{Pt}(6 \mathrm{~nm})$ and Co $(8 \mathrm{~nm})$ layers as well as on $\mathrm{Ta}(1,6 \mathrm{~nm})|\mathrm{Cu}(2,4,6 \mathrm{~nm})| \mathrm{Co}(2.5 \mathrm{~nm})$ trilayers, all capped by $2 \mathrm{~nm} \mathrm{AlO}_{x}$. The USMR is absent in the Ta, Pt and Co single layers, which also excludes any self-induced magnetoresistive effect ${ }^{32}$ and proves the essential role of the FM/NM 


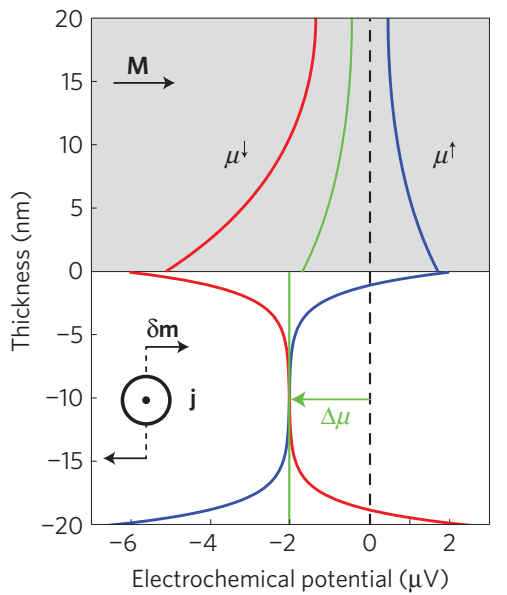

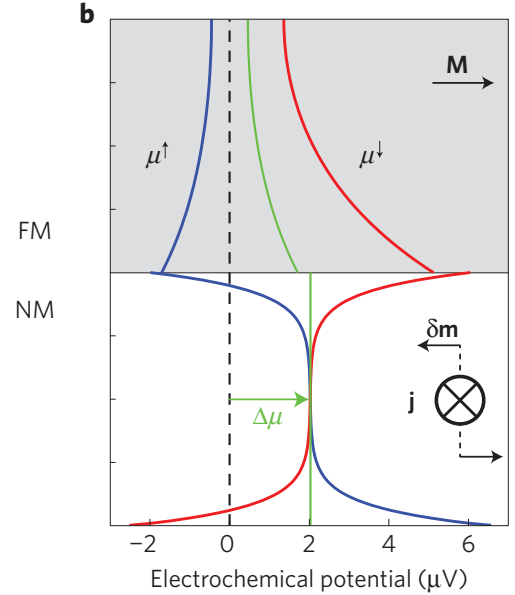

c

$$
\mathbf{M} \times \mathbf{j}<0
$$

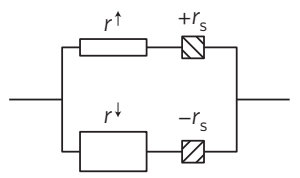

$\mathbf{M} \times \mathbf{j}>0$

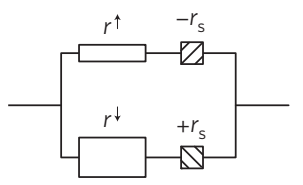

Figure 5 | Modulation of the spin accumulation, spin-dependent electrochemical potential, and interface resistance by the SHE. a,b, Profile of the electrochemical potential of majority ( $\mu^{\uparrow}$, blue lines) and minority ( $\mu^{\downarrow}$, red lines) electrons in the proximity of the FM/NM interface for positive (a) and negative (b) current. The electrochemical potential of the NM shifts relative to that of the FM, as indicated by the green arrow. The direction of the magnetization is $\mathbf{M} \| \hat{\mathbf{y}}$ in both panels. In our notation, the majority spins are oriented antiparallel to $\mathbf{M}$ and $\delta \mathbf{m}$, the non-equilibrium magnetization induced by the SHE, has opposite sign relative to $\boldsymbol{\mu}_{\mathrm{s}}$. Reversing $\mathbf{M}$ is equivalent to exchanging $\mu^{\uparrow}$ and $\mu^{\downarrow}$ and inverting $\Delta \mu$. The sign of the SHE corresponds to $\mathrm{Pt} \mid \mathrm{Co}$; the parameters used to calculate $\mu^{\uparrow}$ and $\mu^{\downarrow}$ are given in the Supplementary Information. c, Two-current series resistor model of the USMR corresponding to $\mathbf{a}$ (top, higher resistance) and $\mathbf{b}$ (bottom, lower resistance). Note that the resistances $r^{\uparrow}$ and $r^{\downarrow}$ may be generalized to include also the bulk spin-dependent resistances of the FM layer.

interface. On the other hand, we find a sizable USMR when a $\mathrm{Cu}$ spacer is inserted between Ta and Co, which excludes proximityinduced magnetization as a possible cause for the USMR (see Supplementary Information).

\section{Discussion}

On the basis of the analysis presented above, we conclude that the current-induced spin accumulation creates an additional spindependent interface resistance that adds to or subtracts from the total resistance depending on the sign of the cross-product $\mathbf{j} \times \mathbf{M}$. Given the in-plane geometry, the interpretation of this effect requires a Boltzmann equation approach to model the spin- and momentum-dependent reflection and transmission of electrons at the FM/NM interface, equivalent to extending the theory of CIP-GMR (refs 29,30) beyond first order and including the SHE. However, a qualitative understanding of the USMR can be derived also by introducing a two-current series resistor model and an interface resistance term proportional to the SHE-induced shift of the electrochemical potential between the FM and NM layers. The latter can be calculated using a one-dimensional drift-diffusion approach $^{9,10,17}$. We consider two separate conduction channels for the minority (spin $\uparrow$ ) and majority (spin $\downarrow$ ) electrons. As in bulk FM, scattering at the interface is spin-dependent due to the unequal density of majority and minority states near the Fermi level, which, in most cases, leads to a larger resistance for minority electrons relative to majority electrons: $r^{\downarrow}>r^{\uparrow}$. This resistance difference is at the heart of the GMR effect, both in the CIP (refs 29,30,33) and the current-perpendicular-to-plane (CPP) geometry ${ }^{10,34}$. Furthermore, when an electric current flows from a FM to a NM or vice versa, another resistance term appears due to the conductivity mismatch between majority and minority electrons on opposite sides of the junction, which results in spin accumulation (refs 8,9). This socalled 'spin-coupled interface resistance' plays a role in CPP-GMR as well as in local and non-local spin-injection devices ${ }^{7,10,35}$, whereas in the CIP geometry it is usually neglected because there is no net charge flow across the interface and the spin accumulation is assumed to be zero. If we take the SHE into account, however, the transverse spin current flowing between the NM and the FM induces a splitting of the spin-dependent electrochemical potentials $\mu^{\uparrow}$ and $\mu^{\downarrow}$ and a net interfacial spin accumulation $\mu_{\mathrm{s}}=\mu^{\uparrow}-\mu^{\downarrow}$, which is given by

$$
\begin{aligned}
\mu_{\mathrm{s}_{\mathrm{N}}}= & \mu_{\mathrm{s}_{\mathrm{N}}}^{0} \tanh \frac{t_{\mathrm{N}}}{2 \lambda_{\mathrm{N}}} \\
& \times \frac{1+\frac{r_{\mathrm{b}}}{\rho_{\mathrm{F}} \lambda_{\mathrm{F}}}\left(1-P^{2}\right) \tanh \frac{t_{\mathrm{F}}}{\lambda_{\mathrm{F}}}}{1+\left(\frac{\rho_{\mathrm{N}} \lambda_{\mathrm{N}}}{\rho_{\mathrm{F}} \lambda_{\mathrm{F}}} \operatorname{coth} \frac{t_{\mathrm{N}}}{\lambda_{\mathrm{N}}}-\frac{r_{\mathrm{b}}}{\rho_{\mathrm{F}} \lambda_{\mathrm{F}}}\right)\left(1-P^{2}\right) \tanh \frac{t_{\mathrm{F}}}{\lambda_{\mathrm{F}}}}
\end{aligned}
$$

where $\mu_{\mathrm{sN}}^{0}=2 e \rho_{\mathrm{N}} \lambda_{\mathrm{N}} \theta_{\mathrm{SH}} j$ is the bare spin accumulation due to the SHE that would occur in a single, infinitely thick NM layer, $\theta_{\mathrm{SH}}$ the spin Hall angle of the NM, $\rho_{\mathrm{N}, \mathrm{F}}$ and $\lambda_{\mathrm{N}, \mathrm{F}}$ are the resistivity and spin diffusion length of the NM and FM, respectively, and $r_{\mathrm{b}}=\left(r^{\uparrow}+r^{\downarrow}\right) / 4$ is the interface resistance ${ }^{10}$. Moreover, the same effect induces a shift $\Delta \mu=\mu_{\mathrm{N}}-\mu_{\mathrm{F}}$ of the electrochemical potential $\mu_{\mathrm{N}, \mathrm{F}}=\left(\mu_{\mathrm{N}, \mathrm{F}}^{+}+\mu_{\mathrm{N}, \mathrm{F}}^{-}\right) / 2$ of the NM relative to the FM:

$$
\begin{aligned}
\Delta \mu_{\mathrm{N}}= & -(P+\gamma \tilde{r}) \mu_{\mathrm{s}_{\mathrm{N}}}^{0} \tanh \frac{t_{\mathrm{N}}}{2 \lambda_{\mathrm{N}}} \\
& \times \frac{1}{1+\tilde{r}} \frac{1}{1+\frac{\frac{\rho_{\mathrm{N}} \lambda_{\mathrm{N}}}{\rho_{\mathrm{F}} \lambda_{\mathrm{F}}}\left(1-P^{2}\right) \tanh \frac{t_{\mathrm{F}}}{\lambda_{\mathrm{F}}} \operatorname{coth} \frac{\mathrm{t}_{\mathrm{N}}}{\lambda_{\mathrm{N}}}}{1-\tilde{r}}}
\end{aligned}
$$

where $\gamma=\left(r^{\downarrow}-r^{\uparrow}\right) /\left(r^{\uparrow}+r^{\downarrow}\right)$ and $\tilde{r}=\left(r_{\mathrm{b}} / \rho_{\mathrm{F}} \lambda_{\mathrm{F}}\right)\left(1-P^{2}\right)$ $\tanh \left(t_{\mathrm{F}} / \lambda_{\mathrm{F}}\right)$. Figure $5 \mathrm{a}, \mathrm{b}$ shows a graphical representation of $\mu_{\mathrm{s}}$ and $\Delta \mu_{\mathrm{N}}$; details about the derivation of equations (2) and (3) are given in the Supplementary Information. A key point is that $\Delta \mu_{\mathrm{N}}$ depends on the product $P \theta_{\mathrm{SH}} j$, as the USMR, and is linked with the spin-dependent scattering potential that gives rise to different transmission coefficients for majority and minority electrons at the FM/NM interface ${ }^{36}$. We can thus draw the following qualitative interpretation of the USMR: when the non-equilibrium magnetization induced by the SHE and the magnetization of the FM are parallel to each other $(\delta \mathbf{m} \| \mathbf{M})$, the transmission of $\uparrow$ $(\downarrow)$ electrons across the interface is reduced (enhanced) by the accumulation of majority electrons at the FM/NM boundary, due to the conductivity mismatch of $\uparrow$ and $\downarrow$ spins in the two materials. Likewise, when $\delta \mathbf{m} \|-\mathbf{M}$, the transmission of $\downarrow$ ( $\uparrow)$ electrons across the interface is reduced (enhanced) since minority 
electrons accumulate at the FM/NM boundary. The overall effect is a modulation of the interface resistance of the $\uparrow$ and $\downarrow$ spin channels by a nonlinear term $\pm r_{s}$, as schematized in Fig. $5 c$. This two-current ( $\uparrow$ and $\downarrow$ ) series resistor model leads to a resistance difference between the two configurations, given by $2 r_{\mathrm{s}} \gamma$, where $r_{\mathrm{s}}$ is assumed proportional to $\Delta \mu_{\mathrm{N}}$.

Accordingly, using realistic values of $r_{\mathrm{b}}, \rho_{\mathrm{N}}$ and $\rho_{\mathrm{F}}$ for $\mathrm{Ta} \mid \mathrm{Co}$ and $\mathrm{Pt} \mid \mathrm{Co}$, we fit the dependence of the USMR on current and NM thickness to the following phenomenological expression (see Supplementary Information): $\Delta R^{\mathrm{USMR}}=A \tanh \left(t_{\mathrm{N}} / 2 \lambda_{\mathrm{N}}\right) /$ $\left(1+R_{\mathrm{FI}} / R_{\mathrm{N}}\right)^{2}$, where $A$ is a parameter proportional to $P \mu_{\mathrm{s}_{\mathrm{N}}}^{0}$ representing the amplitude of the effect, $R_{\mathrm{FI}}$ is the effective resistance of the FM and interface regions, and $R_{\mathrm{N}}=\rho_{\mathrm{N}} l /\left(w t_{\mathrm{N}}\right)$ is the resistance of the NM. The denominator accounts for the decreased fraction of electrons that scatter at the interface as the thickness of the NM increases. Similarly, we obtain $\Delta R^{\mathrm{USMR}} / R=(A / R) \tanh \left(t_{\mathrm{N}} / 2 \lambda_{\mathrm{N}}\right) /\left(1+R_{\mathrm{FI}} / R_{\mathrm{N}}\right)$. As shown in Fig. 4 , these simple expressions fit $\Delta R^{\mathrm{USMR}}$ and $\Delta R^{\mathrm{USMR}} / R$ remarkably well, providing also values of $\lambda_{\mathrm{Pt}}=1.1 \mathrm{~nm}$ and $\lambda_{\mathrm{Ta}}=1.4 \mathrm{~nm}$ that are in agreement with previous measurements ${ }^{18,31}$. Our model thus captures the essential features of the USMR, namely its sign, angular dependence, and proportionality to the current. Detailed calculations including realistic scattering parameters within a nonlinearized Boltzmann approach including the SHE (ref. 37) should be able to account for quantitative aspects of the USMR in different materials. We stress also that the USMR is not uniquely linked to the SHE but may arise also due to other sources of non-equilibrium spin accumulation, such as the Rashba effect at FM/NM interfaces and topological insulators ${ }^{37-40}$, as well as the anomalous Hall effect in FM.

\section{Conclusions}

The existence of a nonlinear magnetoresistive term proportional to $\mathbf{j} \times \mathbf{M}$ has both fundamental and practical implications. Identifying which symmetries survive the breakdown of the Onsager relationships in the nonlinear regime is central to the understanding of electron transport phenomena, particularly in mesoscopic and magnetic conductors, where such effects can also have thermoelectric and magneto-optical counterparts ${ }^{41,42}$. In this respect, the USMR shows that the longitudinal conductivity has an antisymmetric Hall-like component that has so far remained unnoticed. We expect such a component to be a general feature of non-centrosymmetric magnetic systems with strong spin-orbit coupling. We note also that the USMR differs from the nonlinear MR observed in chiral conductors, such as metal helices ${ }^{42}$ and molecular crystals ${ }^{43}$, which is proportional to $\mathbf{j} \cdot \mathbf{M}$.

In the field of spintronics, nonlinear interactions between spin and charge are emerging as a tool to detect spin currents ${ }^{44}$ and thermoelectric ${ }^{45}$ effects, as well as magnetization reversal in dual spin valves ${ }^{46}$. Although the USMR is only a small fraction of the total resistance, its relative amplitude is of the same order of magnitude as the spin transresistance measured in non-local metal spin valves ${ }^{7,35}$, which is a method of choice for the investigation of spin currents. The thermoelectric counterpart of the USMR, related to the spin Nernst effect, may be used to detect heat-induced spin accumulation by modulation of the magnetization rather than an electric current. We note that the electric field created by the USMR is of the order of $2 \mathrm{~V} \mathrm{~m}^{-1}$ per $10^{7} \mathrm{~A} \mathrm{~cm}^{-2}$, which is comparable to the ANE (ref. 23) and three orders of magnitude larger than the typical electric fields due to the SSE (refs 27,28).

In terms of applications, the USMR may be used to add $360^{\circ}$ directional sensitivity to AMR sensors, which are widely employed for position, magnetic field, and current sensing, and already include built-in modulation circuitry for accurate resistance measurements. Most interestingly, the USMR shows that it is possible to realize twoterminal spintronic devices where switching is performed by SOTs (refs 14,15) and reading by a resistance measurement. Such a scheme involves only one FM layer and minimum patterning effort. Finally, we believe that there is substantial room for improving the amplitude of the USMR to levels closer to the AMR, either by material or heterostructure engineering. In particular, the USMR could increase significantly in magnetic topological insulator structures due to the very large spin accumulation and reduced bulk conductance reported for these systems ${ }^{47,48}$.

Note added in proof: After submission of this manuscript, Olejník et al. reported a similar effect in a ferromagnetic/paramagnetic GaMnAs bilayer ${ }^{49}$. This observation nicely confirms our findings in a different material system. Further, it shows that the USMR increases by orders of magnitude in conductors where the charge carrier density is small compared to metals.

\section{Methods}

Methods and any associated references are available in the online version of the paper.

Received 11 February 2015; accepted 6 May 2015; published online 8 June 2015

\section{References}

1. Thomson, W. On the electro-dynamic qualities of metals: Effects of magnetization on the electric conductivity of nickel and of iron. Proc. R. Soc. Lond. 8, 546-550 (1856)

2. Campbell, I., Fert, A. \& Jaoul, O. The spontaneous resistivity anisotropy in Ni-based alloys. J. Phys. C 3, S95-S101 (1970).

3. McGuire, T. \& Potter, R. Anisotropic magnetoresistance in ferromagnetic 3d alloys. IEEE Trans. Magn. 11, 1018-1038 (1975).

4. Baibich, M. N. et al. Giant magnetoresistance of (001)Fe/(001)Cr magnetic superlattices. Phys. Rev. Lett. 61, 2472-2475 (1988).

5. Binasch, G., Grünberg, P., Saurenbach, F. \& Zinn, W. Enhanced magnetoresistance in layered magnetic structures with antiferromagnetic interlayer exchange. Phys. Rev. B 39, 4828-4830 (1989)

6. Johnson, M. \& Silsbee, R. H. Interfacial charge-spin coupling: Injection and detection of spin magnetization in metals. Phys. Rev. Lett. 55, 1790-1793 (1985).

7. Jedema, F., Filip, A. \& Van Wees, B. Electrical spin injection and accumulation at room temperature in an all-metal mesoscopic spin valve. Nature $\mathbf{4 1 0}$ 345-348 (2001)

8. Johnson, M. \& Silsbee, R. Thermodynamic analysis of interfacial transport and of the thermomagnetoelectric system. Phys. Rev. B 35, 4959-4972 (1987).

9. Van Son, P., Van Kempen, H. \& Wyder, P. Boundary resistance of the ferromagnetic-nonferromagnetic metal interface. Phys. Rev. Lett. 58, 2271-2273 (1987).

10. Valet, T. \& Fert, A. Theory of the perpendicular magnetoresistance in magnetic multilayers. Phys. Rev. B 48, 7099-7113 (1993)

11. Maekawa, S., Valenzuela, S. O., Saitoh, E. \& Kimura, T. Spin Current (Oxford Univ. Press, 2012).

12. Brataas, A., Bauer, G. E. \& Kelly, P. J. Non-collinear magnetoelectronics. Phys. Rep. 427, 157-255 (2006).

13. Sinova, J., Valenzuela, S. O., Wunderlich, J., Back, C. H. \& Jungwirth, T. Spin Hall effect. Preprint at http://arxiv.org/abs/1411.3249 (2014).

14. Miron, I. M. et al. Perpendicular switching of a single ferromagnetic layer induced by in-plane current injection. Nature 476, 189-193 (2011).

15. Liu, L. et al. Spin-torque switching with the giant spin Hall effect of tantalum. Science 336, 555-558 (2012).

16. Garello, K. et al. Symmetry and magnitude of spin-orbit torques in ferromagnetic heterostructures. Nature Nanotech. 8, 587-593 (2013).

17. Nakayama, H. et al. Spin Hall magnetoresistance induced by a nonequilibrium proximity effect. Phys. Rev. Lett. 110, 206601 (2013).

18. Hahn, C. et al. Comparative measurements of inverse spin Hall effects and magnetoresistance in YIG/Pt and YIG/Ta. Phys. Rev. B 87, 174417 (2013).

19. Althammer, M. et al. Quantitative study of the spin Hall magnetoresistance in ferromagnetic insulator/normal metal hybrids. Phys. Rev. B 87, 224401 (2013).

20. Miao, B., Huang, S., Qu, D. \& Chien, C. Physical origins of the new magnetoresistance in Pt/YIG. Phys. Rev. Lett. 112, 236601 (2014).

21. Avci, C. O. et al. Fieldlike and antidamping spin-orbit torques in as-grown and annealed Ta/CoFeB/MgO layers. Phys. Rev. B 89, 214419 (2014)

22. Hayashi, M., Kim, J., Yamanouchi, M. \& Ohno, H. Quantitative characterization of the spin-orbit torque using harmonic Hall voltage measurements. Phys. Rev. B 89, 144425 (2014). 
23. Avci, C. O. et al. Interplay of spin-orbit torque and thermoelectric effects in ferromagnet/normal-metal bilayers. Phys. Rev. B 90, 224427 (2014).

24. Kobs, A. et al. Anisotropic interface magnetoresistance in $\mathrm{Pt} / \mathrm{Co} / \mathrm{Pt}$ sandwiches. Phys. Rev. Lett. 106, 217207 (2011).

25. Lu, Y. et al. Hybrid magnetoresistance in the proximity of a ferromagnet. Phys. Rev. B 87, 220409 (2013).

26. Kim, J. et al. Layer thickness dependence of the current-induced effective field vector in $\mathrm{Ta}|\mathrm{CoFeB}| \mathrm{MgO}$. Nature Mater. 12, 240-245 (2013).

27. Weiler, M. et al. Local charge and spin currents in magnetothermal landscapes. Phys. Rev. Lett. 108, 106602 (2012).

28. Kikkawa, T. et al. Longitudinal spin Seebeck effect free from the proximity Nernst effect. Phys. Rev. Lett. 110, 067207 (2013).

29. Camley, R. E. \& Barnaś, J. Theory of giant magnetoresistance effects in magnetic layered structures with antiferromagnetic coupling. Phys. Rev. Lett. 63, 664-667 (1989).

30. Hood, R. Q. \& Falicov, L. Boltzmann-equation approach to the negative magnetoresistance of ferromagnetic-normal-metal multilayers. Phys. Rev. B 46, 8287-8296 (1992).

31. Zhang, W. et al. Determination of the Pt spin diffusion length by spin-pumping and spin Hall effect. Appl. Phys. Lett. 103, 242414 (2013).

32. Dyakonov, M. Magnetoresistance due to edge spin accumulation. Phys. Rev. Lett. 99, 126601 (2007).

33. Dieny, B. Classical theory of giant magnetoresistance in spin-valve multilayers: Influence of thicknesses, number of periods, bulk and interfacial spin-dependent scattering. J. Phys. Condens. Matter 4, 8009-8020 (1992).

34. Nguyen, H., Pratt, W. Jr \& Bass, J. Spin-flipping in Pt and at Co/Pt interfaces. J. Magn. Magn. Mater. 361, 30-33 (2014).

35. Valenzuela, S. O. \& Tinkham, M. Direct electronic measurement of the spin Hall effect. Nature 442, 176-179 (2006)

36. Zhang, S., Levy, P. \& Fert, A. Conductivity and magnetoresistance of magnetic multilayered structures. Phys. Rev. B 45, 8689-8702 (1992).

37. Haney, P. M., Lee, H-W., Lee, K-J., Manchon, A. \& Stiles, M. Current induced torques and interfacial spin-orbit coupling: Semiclassical modeling. Phys. Rev. $B$ 87, 174411 (2013).

38. Manchon, A. \& Zhang, S. Theory of nonequilibrium intrinsic spin torque in a single nanomagnet. Phys. Rev. B 78, 212405 (2008).

39. Miron, I. M. et al. Current-driven spin torque induced by the Rashba effect in a ferromagnetic metal layer. Nature Mater. 9, 230-234 (2010).

40. Mahfouzi, F., Nagaosa, N. \& Nikolić, B. K. Spin-orbit coupling induced spin-transfer torque and current polarization in topological-insulator/ferromagnet vertical heterostructures. Phys. Rev. Lett. 109, 166602 (2012).
41. Sánchez, D. \& Büttiker, M. Magnetic-field asymmetry of nonlinear mesoscopic transport. Phys. Rev. Lett. 93, 106802 (2004).

42. Rikken, G., Fölling, J. \& Wyder, P. Electrical magnetochiral anisotropy. Phys. Rev. Lett. 87, 236602 (2001).

43. Pop, F., Auban-Senzier, P., Canadell, E., Rikken, G. L. \& Avarvari, N. Electrical magnetochiral anisotropy in a bulk chiral molecular conductor. Nature Commun. 5 (2014).

44. Vera-Marun, I. J., Ranjan, V. \& van Wees, B. J. Nonlinear detection of spin currents in graphene with non-magnetic electrodes. Nature Phys. 8, 313-316 (2012).

45. Slachter, A., Bakker, F. L., Adam, J-P. \& van Wees, B. J. Thermally driven spin injection from a ferromagnet into a non-magnetic metal. Nature Phys. 6, 879-882 (2010).

46. Aziz, A. et al. Nonlinear giant magnetoresistance in dual spin valves. Phys. Rev. Lett. 103, 237203 (2009).

47. Mellnik, A. et al. Spin-transfer torque generated by a topological insulator. Nature 511, 449-451 (2014).

48. Fan, Y. et al. Magnetization switching through giant spin-orbit torque in a magnetically doped topological insulator heterostructure. Nature Mater. 13, 699-704 (2014).

49. Olejník, K., Novák, V., Wunderlich, J. \& Jungwirth, T. Electrical detection of magnetization reversal without auxiliary magnets. Phys. Rev. B 91, 180402(R) (2015).

\section{Acknowledgements}

This work was funded by the Swiss National Science Foundation (Grant No. 200021-153404) and the European Commission under the 7th Framework Program (SPOT project, Grant No. 318144)

\section{Author contributions}

C.O.A., K.G. and P.G. planned the experiments; M.G., A.G., S.F.A. and C.O.A. carried out the sample growth and patterning; C.O.A., K.G. and A.G. performed the measurements; C.O.A. and P.G. analysed the data and wrote the manuscript. All authors contributed to the discussion of the data in the manuscript and Supplementary Information.

\section{Additional information}

Supplementary information is available in the online version of the paper. Reprints and permissions information is available online at www.nature.com/reprints.

Correspondence and requests for materials should be addressed to C.O.A. or P.G.

\section{Competing financial interests}

The authors declare no competing financial interests. 


\section{Methods}

Sample preparation. The $\mathrm{Pt}(1-9 \mathrm{~nm})|\mathrm{Co}(2.5 \mathrm{~nm})| \mathrm{AlO}_{x}(2 \mathrm{~nm})$ and $\mathrm{Ta}$ $(1-9 \mathrm{~nm})|\mathrm{Co}(2.5 \mathrm{~nm})| \mathrm{AlO}_{x}(2 \mathrm{~nm})$ layers were grown by d.c. magnetron sputtering on thermally oxidized $\mathrm{Si}$ wafers. The deposition rates were $0.185 \mathrm{~nm} \mathrm{~s}^{-1}$ for $\mathrm{Pt}$, $0.067 \mathrm{~nm} \mathrm{~s}^{-1}$ for Ta, $0.052 \mathrm{~nm} \mathrm{~s}^{-1}$ for Co, and $0.077 \mathrm{~nm} \mathrm{~s}^{-1}$ for Al. The deposition pressure was $2 \mathrm{mtorr}$ and the power was $50 \mathrm{~W}$ for all targets. The $\mathrm{Al}$ capping layers were oxidized in situ by a $7 \mathrm{~m}$ torr $\mathrm{O}_{2}$ plasma at $10 \mathrm{~W}$ for $35 \mathrm{~s}$. The layers were subsequently patterned into six-terminal Hall bars by means of standard optical lithography and Ar milling procedures. The Hall bar dimensions are $w$ for the current line width, $w / 2$ for the Hall branch width, with $l=4 w$ being the distance between two Hall branches, where $w$ varies between 4 and $10 \mu \mathrm{m}$.

Characterization. All layers possess spontaneous isotropic in-plane magnetization. To determine the saturation magnetization of Co we have performed anomalous Hall effect measurements on an 8-nm-thick Co reference sample with $B_{\text {ext }} \| \mathbf{z}$. The field required to fully saturate $\mathbf{M}$ out-of-plane is about $1.5 \mathrm{~T}$; which, assuming that perpendicular magnetic anisotropy is negligible in this layer, is close to $\mu_{0} M_{s}$ expected of Co. Similar measurements on $\mathrm{Ta}(6 \mathrm{~nm}) \mid \mathrm{Co}(2.5 \mathrm{~nm})$ and $\mathrm{Pt}(6 \mathrm{~nm}) \mid \mathrm{Co}$ $(2.5 \mathrm{~nm}$ ) layers give saturation fields of $1.45 \mathrm{~T}$ and $0.8 \mathrm{~T}$, respectively. This is attributed to the small (large) perpendicular interface anisotropy contribution of the $\mathrm{Ta} / \mathrm{Co}(\mathrm{Pt} / \mathrm{Co})$ interface, reducing the field required to saturate the magnetization out-of-plane. Four-point resistivity measurements on single Co $(8 \mathrm{~nm}), \mathrm{Ta}(6 \mathrm{~nm})$ and $\mathrm{Pt}(6 \mathrm{~nm})$ layers yield $\rho_{\mathrm{Co}}=25.3 \mu \Omega \mathrm{cm}, \rho_{\mathrm{Ta}}=237 \mu \Omega \mathrm{cm}$ and $\rho_{\mathrm{Pt}}=34.1 \mu \Omega \mathrm{cm}$, in line with expectations for Pt and Co thin films, and the $\beta$-phase of Ta. The magnetoresistance and Hall voltage measurements were performed at room temperature using an a.c. current $I=I_{0} \sin \omega t$, where $\omega / 2 \pi=10 \mathrm{~Hz}$, generated by a Keithley-6221 constant current source. For the data reported in Fig. 2 the peak amplitude of the injected a.c. current was set to $8.5 \mathrm{~mA}$ corresponding to a nominal current density of $j=10^{7} \mathrm{~A} \mathrm{~cm}^{-2}$. In other measurements with different device size and thickness, the current was adapted to have the same current density. The longitudinal and transverse voltages were recorded simultaneously by means of a 24-bit resolution National Instruments PXI-4462 dynamic signal analyser, dwelling $10 \mathrm{~s}$ at each angle position in a uniform external field of $1.7 \mathrm{~T}$. The rotation of the sample was provided by a motorized stage with a precision of $0.02^{\circ}$. The acquired voltages were fast Fourier transformed to extract the first and second harmonic voltage signals $V_{\omega}$ and $V_{2 \omega}$. The corresponding resistances are given by $R_{\omega}=V_{\omega} / I_{0}$ and $R_{2 \omega}=V_{2 \omega} / I_{0}$ (peak values). 